\title{
Información a la población en situaciones de emergencia y riesgo colectivo
}

\section{Information to population in emergency situations and collective hazard situations}

M. ${ }^{\text {a }}$ Patricia ACINAS ACINAS*

\section{RESUMEN}

Este artículo revisa el proceso de comunicación pública en emergencias. Un mensaje de alerta puede facilitar a la población información sobre riesgos inminentes que han precipitado la alerta de emergencia.

Informar a la población sobre las características del riesgo minimizará la probabilidad de que las personas malinterpreten el riesgo y tomen decisiones incorrectas respecto a qué hacer, especialmente en emergencias prolongadas. La información no conduce al pánico. Además informar a la población sobre los riesgos puede incrementar la confianza en las autoridades de emergencias locales y puede prepararles para enfrentarse a los riesgos lo más pronto posible.

La respuesta humana a las alertas por riesgos está influenciada por varios factores. Si las personas responsables los conocen, podrian hacer cambios en futuros planes de evacuación.

Aunque la sabiduría popular considera que las falsas alarmas reducen la disponibilidad de la población para responder a futuros eventos, algunas investigaciones han hallado que esto puede mostrar cómo los humanos responden a alertas y lo que deben hacer los Directores de Emergencia respecto a la gestión de la emergencia

\section{PALABRAS CLAVE}

Información, Comunicación, Conducta colectiva, Emergencia, Riesgo, Respuesta de alerta.

*Psicóloga. Especialista en Psicología de Urgencias, Emergencias y Catástrofes. Supervisora Nacional de IPSE Intervención Psicológica Especializada. pacinas@ipse-psicologia.com 
Información a la población en situaciones de emergencia y riesgo colectivo

\section{ABSTRACT}

This article reviews the process of public response to emergencies. A warning message must provide the public with information about the impending hazard that has precipitated the emergency warning.

Informing the population about characteristics of the hazard will minimise the likehood of people misperceiving the hazard and making incorrect decisions about what to do, especially in protracted emergencies. Information does not lead to panic. Also, informing the public about risks can increase trust in local emergency authorities and it can prepare people to deal with all the risks as soon as possible.

The human response to warnings for hazards is affected by many factors. If the people in charge know them they would make few changes in future evacuation plans.

Although conventional wisdom is that false alarms reduce the public's willingness to respond to future events, some research has obtained that it can show how humans respond to warnings and what have to do Emergency Managers about conducting an emergency.

\section{KEY WORDS}

Information, Communication, Collective behaviour, Emergency, Hazard, Warning response. 


\section{INTRODUCCIÓN}

Las situaciones de emergencia se pueden producir de manera inesperada; ninguna persona cree que le vaya a suceder nada en el lugar en que vive, a ella o a las personas cercanas; pero las emergencias ocurren y nos afectan a todos de manera directa o indirecta.

Por otro lado para las Administraciones públicas y para grandes empresas que pueden resultar afectadas el saber qué decir, cómo, cuándo, quién, a quién, de qué manera, empleando qué medio o qué soporte... puede convertirse en una situación dificil si no se cuenta con una previsión o un esquema de actuación claro. Si además hay muchas personas implicadas y sus respectivos familiares, a quienes dar información en esos momentos, la situación se complica considerablemente.

A pesar de la modernización y especialización creciente en este campo, queda todavia avanzar más en los aspectos psicosociales de la información (Muñoz y Álvarez, 2000): qué información dar en situaciones de grandes emergencias y/o cuando hay un gran número de personas implicadas en la misma. Esto es fundamental porque en estas situaciones una decisión acertada en cuanto a qué decir o hacer, puede tener consecuencias favorables tanto a nivel de gestión como de percepción de atención por parte de las personas afectadas.

Han sido muchos los lugares del mundo que, en el siglo XX, han experimentado una situación de riesgo colectivo en la que ha sido necesario transmitir información a la población; la colisión de dos aviones en el aeropuerto de Los Rodeos en Tenerife (España) en marzo de 1977 que arrojó 582 víctimas, la catástrofe de Bophal (India), en agosto de 1985; la catástrofe de Chernobyl (en la actual Ucrania) en abril de 1986, las acciones del huracán Katrina en EE.UU en 2005... Generalmente, cuando la situación es grave las autoridades toman medidas al respecto, por ejemplo el accidente en una planta quimica en la localidad de Seveso cercana a Milán el 10 de julio de 1976, que originó la emisión de sustancias tóxicas y graves daños en cultivos, paisaje y medio ambiente de la zona, además de daños y secuelas físicas y psicológicas en las personas a causa de la dioxina. El impacto fue tan grande que se creó en 1988 una Directiva llamada Seveso $(88 / 610 /$ EEC), para la prevención de riesgos tecnológicos, por la que las empresas cuya actividad quimica pueda ocasionar riesgos en la población están obligadas a informar a los ciudadanos.

En España se han dado algunas situaciones en las que la gestión informativa influyó en la evolución de la situación: el incidente producido en la central nuclear Vandellós I de Tarragona en 1989, por un fallo mecánico en el generador eléctrico que dejó inoperativos algunos sistemas de seguridad, cambió las percepciones de la población general sobre el uso de la energía nuclear y los riesgos asociados a la misma, especialmente para personas residentes en emplazamientos cercanos. La población comenzó a recibir mensajes a favor del uso de la energía nuclear (por parte del Consejo de Seguridad Nuclear) y en contra ( reactivación el movimiento antinuclear con manifestaciones públicas en las calles), después de la emergencia; las personas tuvieron la información tiempo después de que se produjera la situación y la percepción de riesgo para su integridad física se formó después del incidente, cuando lo deseable es que tenga lugar antes o durante la situación; la riada del camping Virgen de las Nieves de Biescas en agosto de 1996 (hasta que se divulgó que el camping 
estaba situado en el antiguo curso natural de un rio, los medios de comunicación atribuian la responsabilidad de lo sucedido a otros factores); la autoevacuación voluntaria de cuatro municipios gallegos por el incidente maritimo en el transporte de sustancias quimicas peligrosas, que provocó el embarrancamiento del buque panameño Casón en la Costa de la Muerte gallega, el 5 de diciembre de 1987; en este caso los mensajes de las Autoridades aconsejaban calma y tranquilidad, mientras los rumores de peligrosidad de la carga en los medios de comunicación aumentaban (CEISE, 1989).

O la repercusión mediática que produjo el naufragio del buque Prestige en aguas gallegas en noviembre de 2002, calificada como la mayor catástrofe ecológica en la historia de España; conllevó diferencias técnicas en su manejo y el tratamiento informativo como situación de emergencia que podría afectar a un elevado número de núcleos poblacionales, varió según el medio informativo, provocando cierto desconcierto entre la opinión pública.

En una situación de emergencia el acceso a los recursos de ayuda en emergencia debe ser rápido y precoz (Muñoz y Alvarez, 2000), para minimizar daños y situaciones que si evolucionan pueden hacerlo de manera consistente y desfavorable.

Otra situación reseñable tuvo lugar en julio de 1978, con la explosión en un transporte de mercancias peligrosas que provocó un gran incendio; se destruyó completamente el área del camping de los Alfaques (Dunas de Arena), situado en la localidad de San Carlos de la Rápita (Tarragona). En ese momento había casi 800 turistas; 215 personas encontraron la muerte. A partir de entonces los transportes de mercancias peligrosas no podrían pasar cerca de zonas especialmente concurridas y se divulgó esta información a nivel nacional.

\section{¿QUÉ ES UNA SITUACIÓN DE EMERGENCIA COLECTIVA?}

No hay muchos estudios sobre cómo se comportan las personas en situaciones de emergencia. Desde la psicologia social (Latané y Darley, 1970) se ha estudiado, por ejemplo, el efecto espectador: Consiste en que cuanto mayor es el número de personas que presencia una situación de emergencia, menor es la probabilidad de que cada uno de ellos ayude. Así, cuando se produce una emergencia real, es más probable ser ayudado si hay una sola persona que si hay varias. Las explicaciones son variadas; cuando hay varias personas se producen algunos fenómenos como:

1. La Ignorancia pluralista ("Si nadie ha hecho nada es que realmente no pasa nada; $y$ ahora no voy a hacer yo el ridiculo llamando cuando no ocurre nada").

2. Dilución de la responsabilidad ("Somos muchos; ya habrá llamado alguien y sino ya lo hará alguna persona"). La difusión de la responsabilidad se distribuye entre todos los espectadores.

3. La ambigüedad de la situación: no se sabe lo que ha pasado.

4. La aprensión a la evaluación: miedo a que evalúen nuestra actuación, juzgando si hacemos bien o mal.

Este fenómeno se empezó a estudiar en los años 60 a raíz de casos de espectadores pasivos ante crímenes urbanos 
en situaciones en las que es factible conseguir ayuda, como el asesinato de Kitty Genovese en Nueva York.

Esto es lo que ocurre cuando la persona presencia una urgencia que no le afecta directamente. Pero cuando es la propia persona la que sufre la emergencia, es importante para su resolución que tenga una percepción clara de que "hay salida" (Fidalgo, 2006a) y se pueden entablar acciones encaminadas a gestionar la situación. Si esto no es así, se pueden desatar estados emocionales de indefensión, conductas autoagresivas, paralizantes 0 ataques a otros en el intento de buscar y alcanzar la salida. Muchos de los incendios que se han producido en discotecas (Discoteca Alcalá 20 en Madrid en 1983; discoteca Cromagnon en Buenos Aires en la Nochevieja del año 2004, con 192 fallecidos y más de mil heridos, donde además las salidas de emergencia estaban bloqueadas) explican claramente este fenómeno.

Las primeras reacciones en una situación de emergencia van a estar determinadas por una serie de variables. Fidalgo (2006a) lo sintetiza en las siguientes:

- Forma en la que se informa de la emergencia.

- Grado de gravedad supuesto a la situación.

- Grado de conocimiento del lugar que tiene la persona.

- Grado de entrenamiento en situaciones similares.

- Características del espacio en el que se encuentra.

- Existencia de salidas de socorro y si éstas son visibles.
- Presencia de otras personas y conocimientos de éstas sobre cómo afrontar la situación.

- Experiencias anteriores en situaciones similares (pueden ser vicarias).

- Manifestaciones visibles de la emergencia (humo, llamas, derrumbamiento por seísmo...)

- Características individuales (edad, sexo...)

El mito sobre reacciones de pánico en las situaciones de catástrofe sigue estando presente. Algunos estudios (De Nicolás y Cols., 2000; Robles y Medina, 2002) han mostrado que cerca del $75 \%$ de las personas actúa de manera instintiva y automática, siguiendo modelos de reacción individuales y arraigados. Pero como han corroborado estos mismos autores, sólo una minoría, entre el 10\% y el 20\% desarrollan un fuerte pánico o reacciones de ansiedad; un porcentaje similar (15\%) de personas permanece en calma y mantiene el control.

Por otro lado, las conductas de éxodo rápido sin una indicación de la autoridad que gestiona la emergencia, tienen más inconvenientes que beneficios. San Juan (2001) indica que este tipo de conductas son una variante de las reacciones de huida colectiva, fruto del miedo y la precipitación.

Cuando se produce una situación de peligro que amenaza la vida, se producen dos reacciones habituales ante los hechos ambiguos (Cortés, 2002): Ignorar e investigar. En una situación de incendio pueden darse reacciones como minimización inicial del peligro, comportamiento de huida condicionado por el humo, formación de redes de apoyo entre familiares y conocidos, así como 
otras conductas diferenciales en función del sexo y la edad.

Podríamos decir, siguiendo a Mileti y Peek (2000), que las reacciones de las personas en situaciones de emergencia están influenciadas por sus pensamientos relevantes en esa situación, la comprensión de la misma, las expectativas basadas en experiencias pasadas en similares situaciones (personales o vicarias), los indicios de los distintos eventos que se están produciendo, incluida la conducta de las otras personas en su entorno inmediato... La forma en que se interrelacione todo lo anterior va a afectar a la respuesta colectiva (Turner, Nigg, Paz \& Young, 1981).

Tras las manifestaciones conductuales desencadenadas por la emergencia, las acciones que llevan a cabo las personas más frecuentemente se pueden agrupar en:

1. Evacuación: salir del lugar y ayudar a evacuar a otras personas.

2. Combatir el suceso con los medios disponibles en ese momento o lugar.

3. Dar el aviso de alarma, ir a ver lo que está pasando.

4. Prevenir a los demás de lo sucedido.

\section{Reunirse con otras personas.}

Pero en una situación de emergencia, además de reacciones individuales, también hay manifestaciones conductuales de tipo colectivo, que ejercen influencia en todas las personas implicadas en la misma. El fenómeno de la conducta colectiva, conducta de masas o dinámica colectiva, se puede definir como (Fidalgo, 2006b), toda conducta relativa- mente espontánea ejecutada por un grupo de personas, ante un estímulo común, en una situación indefinida o ambigua. Estos grupos de personas son generalmente transitorios y carentes de organización formal, y reaccionan ante un conjunto inmediato de circunstancias de formas no convencionales.

En una situación de riesgo, la respuesta de la población puede ser adaptada, (con lo que es más fácil dirigirse a ella) o desadaptada (lo que complica enormemente la gestión de la situación). Cuando se observan conductas colectivas adecuadas (como el orden en la evacuación de una población de riesgo), se puede luchar contra la propagación del peligro (o los rumores) de manera temprana y realizar una organización racional de los recursos. Pero cuando se observan conductas inadecuadas (como considerar que la situación es irreal o éxodos masivos sin control) se aumenta también la exposición al peligro (San Juan, 2001).

Por otro lado las reacciones también se pueden reproducir o contagiar de unas personas a otras, de una manera no prevista inicialmente. Conviene analizar los posibles factores que han podido conducir a este contagio antes de realizar ninguna intervención.

Además los propios intervinientes se pueden ver influenciados por las dificultades de comunicación a nivel intraorganizacional como inter-organizacional; lo cual puede complicar la actuación y saber qué es lo que debe hacerse realmente. Por ejemplo si no hay acuerdo respecto al lugar al que hay que evacuar a las víctimas o por dónde se debe rescatar a varias personas atrapadas...

Otro aspecto importante es que el potencial de crisis psicológica en estas circunstancias, puede surgir en los días 
y semanas inmediatamente posteriores, cuando los individuos tratan de enfrentarse a las pérdidas (Slaikeu, 1996).

Podemos concretar una serie de conductas de riesgo en situación de emergencia que pueden minimizarse con un adecuado manejo de la información.

1. No seguir las indicaciones porque las personas necesitan buscar encontrar 0 asegurarse de que sus seres queridos están bien.

2. Retroceder en una evacuación porque han olvidado un objeto muy preciado o con valor sentimental.

3. Tardar en abandonar un lugar, con riesgo de muerte inminente (para la persona y/o sus allegados) por querer coger algo que se considera imprescindible.

4. Minusvalorar el/los riesgo/s comunicados por la autoridad competente.

5. No respetar prohibiciones o restricciones (a corto o largo plazo) estipuladas de uso de ciertos servicios (agua, vehículos...)

6. No querer acceder a la información periódica proporcionada, por miedo, rebeldia, negación del riesgo emergente.

7. Considerar que las autoridades están realizando acciones suficientes para solucionar el incidente y pensar que sólo se podrá arreglar con sus intervenciones, porque la persona considera que no tiene nada que hacer (afrontamiento pasivo). La organización ORAU (2006) habla de implicar a las personas como participantes y no como espectadores de lo sucedido, para facilitar la gestión de la emergencia.

8. Creer que la situación está controlada, cuando realmente no lo está (y puede que tarde en estarlo). Transmitir a otros esta percepción con el consiguiente riesgo para todos.

9. Poseer dificultades para la comprensión del mensaje (distinto idioma, diferente nivel cultural, interferencias o cortes en el mensaje debido a los medios técnicos que emiten la comunicación, hipoacusia, presbicia...) y no poner medios para solucionarlo.

10. Dar prominencia a reacciones instintivas frente a las racionales (Por ejemplo, en un incendio, salir corriendo a toda costa empujando a la gente, frente a salir de manera ordenada por los lugares indicados).

11. Querer ayudar a otros sin disponer de los medios técnicos (por ejemplo, oxígeno en un edificio en llamas), ni las habilidades físicas ni psicológicas pertinentes (Por ejemplo, intentar rescatar a un ahogado sin saber nadar).

12. Retrasar la comunicación de la alerta al teléfono de emergencias 112 , al responsable de seguridad/ emergencias de la organización; esto origina una tardanza en tomar medidas precoces y en transmitir a los grupos afectados la gravedad de la situación.

13. Avisar a otras personas de lo ocurrido (medios de comunicación incluidos), con el consiguiente colapso de las líneas telefónicas. Varias investigaciones han encon- 
Información a la población en situaciones de emergencia y riesgo colectivo

trado que esta necesidad es perentoria (alrededor del $76 \%$ de la población lo hace), tanto en el 11 de septiembre (Schuster, Stein, Jaycox, Collins, Marshall, Elliot et al. 2001) como en el 7 de julio en Londres (Rubin, Brewin, Greenberg, Simpson \& Wessely, 2005)

14. Retrasar las acciones tendentes a la seguridad, por considerar que se dispone de tiempo suficiente para llevarlas a cabo.

15. Transmitir un mensaje ambiguo, incompleto, incierto, sin datos comprobados... a otras personas, lo que puede alertarlas innecesariamente o hacer que asuman conductas de mayor riesgo.

16. No proporcionar información completa de las vías de evacuación y/o comunicación afectadas y las seguras (carreteras, calles principales, vías férreas, salidas de emergencia...). En el reciente incendio y vertido tóxico en Candas de Reus en Pontevedra (septiembre de 2006) algunas personas no hicieron caso a los mensajes que indicaban que no se podía circular por algunas carreteras y resultaron atrapados con pocas posibilidades de recibir ayuda.

17. Establecer culpables entre algunos de los sectores de la población afectada, en los primeros momentos; puede dar lugar a dificultades en la gestión de la emergencia y en la interpretación de la información facilitada, aparición de rumores...

18. Otras conductas de riesgo derivadas de las características concretas de la situación de emergencia.
Todas estas conductas debemos considerarlas cuando elaboremos un mensaje para la población, para controlarlas, minimizarlas o canalizarlas adecuadamente, sin que produzcan interferencias en las tareas que exige la gestión eficaz de la emergencia.

\section{SITUACIONES Y RIESGOS ASOCIADOS}

No se han hecho muchas investigaciones relacionadas con situaciones de riesgo, comportamiento de la población e información facilitada en esos momentos; algunos de los estudios que se han hecho han tenido lugar en EE.UU.

Las situaciones en que puede plantearse la necesidad de dar mensajes a la población, son muy variadas (naturales 0 producidas por el hombre, intencionada o no intencionadamente): nevadas, terremotos, riadas, tsunamis, maremotos, incendios, accidentes industriales, alteraciones de la seguridad ciudadana, avisos de bomba, emergencias radiológicas, emisiones (a la atmósfera) o vertidos (liquidos a ríos, mares...) de sustancias químicas peligrosas, derrumbamientos con atrapados, accidentes de ferrocarril, metro, de tráfico con múltiples víctimas, secuestros de aviones, amenazas terroristas en aeropuertos (como la producida el 10 de agosto de 2006 en Heathrow, en Londres, que obligó a cerrar al tráfico aéreo durante casi dos dias, por alerta terrorista máxima, uno de los mayores aeropuertos del mundo y que ha hecho que desde entonces se extremen las medidas de seguridad en controles de aeropuertos respecto a la introducción de envases con liquidos; la red terrorista pretendia hacer estallar aviones en pleno vuelo), etc.

Según Muñoz (2000), podemos distinguir tres tipos de emergencias en función de su temporalidad: 
1. Previsibles (pueden ocurrir, pero no se sabe si tendrán lugar 0 no). Por ejemplo las inundaciones y desbordamientos en grandes ríos de Centroeuropa en el verano de hace unos años; se habían producido situaciones similares hacía mucho tiempo y ese año las lluvias no se produjeron en los mismos periodos ni de la misma forma que en años anteriores.

2. Previstas (se sabe que van a producirse e incluso se puede estimar en qué momento). Por ejemplo el desbordamiento de un río que ocurre todos lo años y obliga a evacuar a una parte de la población.

3. Acaecidas (ocurren de manera brusca e inesperada y no se puede establecer ningún tipo de previsión sobre ellas). Por ejemplo la inundación del Camping de Biescas el 7 de agosto de 1996.

También hay que tener en cuenta que los procesos de los que hablamos se pueden desencadenar en lugares que ya cuentan con riesgos especificos: salas de espectáculos, estadios deportivos, grandes locales (discotecas, sótanos, garajes, hipermercados, hospitales, hoteles, edificios de gran altura...), grandes manifestaciones, huelgas, disturbios callejeros...

En dos de los más graves incendios de nuestro país en lugares públicos, que han implicado un alto número de víctimas, las dificultades más importantes aparecieron por los riesgos asociados. Cortés (2002) indica que en el incendio de la discoteca Alcalá 20, en el año 1983, la complejidad del diseño arquitectónico con varias plantas de sótanos hizo que fallecieran 81 personas y que las tareas de búsqueda y rescate se prolongaran durante cinco días. En 1987 el incendio de Almacenes Arias, en pleno centro de
Madrid, se produjo por el derrumbamiento de un edificio intercomunicado con otro; a pesar de que la evacuación de clientes y empleados fue ordenada, fallecieron 10 bomberos cuando el inmueble se desplomó de manera fortuita sobre ellos en la madrugada del día siguiente; las tareas de rescate y desescombro duraron cuatro días. La información de la que disponían sobre el estado real de la estructura del edificio hizo que no se pudieran tomar en cuenta los riesgos inherentes y que se produjera el triste desenlace. Este incidente consiguió que cambiara la legislación en materia de protección y rescate en incendios.

Las comunicaciones van a variar en función de la situación de emergencia: urgencia, emergencia, simulacro, exhibición...

Cada situación va a requerir unas especificaciones distintas en cuanto a información, recursos materiales y humanos... para favorecer que la situación se resuelva de la mejor manera posible. Por eso es conveniente implementar un sistema de gestión de la emergencia.

Para que sea eficaz, un sistema de alerta debe poseer tres componentes básicos (Sorensen, Vogt \& Mileti, 1987): un subsistema de detección, un subsistema de gestión de la emergencia y un subsistema de respuesta pública a la emergencia. Son tres pasos debidamente coordinados para hacer frente a una situación de peligro inminente, mediante un órgano de gestores de la emergencia que le dé el significado preciso y decida qué tipo de actuación es necesaria en cada caso. Este proceso debe ser interactivo como también añade Mileti y Peek (2000)

La prevención debe ir encaminada a la preparación de la población ante posi- 
Tabla 1: Diferenciación entre simulacro y exhibición (Acinas, 2007)

\begin{tabular}{|c|c|c|}
\hline & SIMUI.ACRO & EXHIBICIÓN \\
\hline Objetivo & $\begin{array}{l}\text { Ensayar una actuación que haya que } \\
\text { llevar a cabo previsiblemente. } \\
\text { Familiarizarse con procedimientos de } \\
\text { actuación que garanticen la seguridad en } \\
\text { situaciones reales. } \\
\text { Identificar riesgos no detectados } \\
\text { previamente y actuar sobre ellos. }\end{array}$ & $\begin{array}{l}\text { Mostrar despliegue de medios técnicos y } \\
\text { humanos con los que se cuenta. } \\
\text { Enseñar las posibilidades de actuación } \\
\text { ante una hipotética situación de } \\
\text { emergencia complicada }\end{array}$ \\
\hline Acciones & LO QUE SE IIACE & LO QUE SE PUEDE IIACER \\
\hline Implicados & $\begin{array}{l}\text { Todos los que pueden verse sorprendidos } \\
\text { por una situación de peligro. } \\
\text { No profesionales y a veces algunos } \\
\text { profesionales. }\end{array}$ & $\begin{array}{l}\text { Los que deben estar preparados para una } \\
\text { situación de emergencia. } \\
\text { Profesionales. }\end{array}$ \\
\hline Situaciones & $\begin{array}{l}\text { Situaciones reales } \\
\text { Riesgos posibles }\end{array}$ & $\begin{array}{l}\text { Situaciones hipotéticas. } \\
\text { Riesgos futuribles. }\end{array}$ \\
\hline $\begin{array}{l}\text { Criterios de } \\
\text { evaluación }\end{array}$ & $\begin{array}{l}\text { Elicacia de la acción. } \\
\text { Rapidez y seguridad. } \\
\text { Aprendizaje ante la situación crítica }\end{array}$ & $\begin{array}{l}\text { Vistosidad, cspectacularidad. } \\
\text { Conocimiento por parte de la población. }\end{array}$ \\
\hline Aviso previo & $\begin{array}{l}\text { No avisar especílicamente (día y hora } \\
\text { concretos) ni comentar las características } \\
\text { del mismo, para valorar conductas } \\
\text { adaptadas a la realidad, si se produjera. }\end{array}$ & $\begin{array}{l}\text { Avisar especílicamente, incluso a medios } \\
\text { de comunicación, para que haya } \\
\text { conocimiento de antemano sobre lo que } \\
\text { se va a hacer. }\end{array}$ \\
\hline Espectadores & $\begin{array}{l}\text { La presencia de espectadores puede } \\
\text { distorsionar las acciones e impedir la } \\
\text { adecuada cjecución. }\end{array}$ & $\begin{array}{l}\text { No se concibe una exhibición si no es } \\
\text { para ser vista por un grupo de } \\
\text { espectadores más o menos numeroso. }\end{array}$ \\
\hline Lugar & $\begin{array}{l}\text { Siempre en el lugar en que puede } \\
\text { producirse la situación de peligro }\end{array}$ & $\begin{array}{l}\text { En el lugar o en instalaciones especiales. } \\
\text { Puede ir acompañado de exposición de } \\
\text { materiales. }\end{array}$ \\
\hline $\begin{array}{l}\text { Relación con } \\
\text { actividades } \\
\text { formativas }\end{array}$ & $\begin{array}{l}\text { Puede ser un paso previo para impartir } \\
\text { una acción formativa. }\end{array}$ & $\begin{array}{l}\text { Suele ser el paso final de una acción } \\
\text { formativa, y/o un medio de evaluación de } \\
\text { la misma. }\end{array}$ \\
\hline
\end{tabular}

bles amenazas, y por otro lado a la formación de los profesionales que intervienen en catástrofes (Fernández Millán, 2005).

\section{ASPECTOS FUNDAMENTALES DE LA GESTIÓN INFORMATIVA DE LA EMERGENCIA}

Autores como la californiana Bourque (2006) informan que la politica de gestión y recursos en desastres ha sido más reactiva que proactiva. Esto ha podido originar una falta de previsión y adaptación previa a las circunstancias de emer- gencia y una disminución en la preparación de la población afectada. Cuando hay una preparación previa es más fácil que las personas adopten las medidas sugeridas.

Se han realizado estudios sobre la influencia de tener información previa a la emergencia. En una investigación tras los atentados de 7 de julio de 2005 en Londres (Rubin, Brewin, Greenberg, Simpson \& Wessely, 2005) se estudió el impacto en la población de las acciones terroristas. Los medios de comunicación del Reino Unido no proporcionaron mucha información a la opinión pública 
(en comparación con los atentados del 11 de septiembre en Nueva York y del 11 de marzo en Madrid); sin embargo antes de los ataques de Londres la población había recibido información en agosto del 2004 (HM Government UK, 2005) sobre qué hacer ante un eventual ataque terrorista, por lo que se estima que la población de Londres estaba en parte preparada para lo que ocurrió. También los mandos políticos y responsables de seguridad estaban advertidos sobre un posible ataque terrorista islámico. Por lo que según estos autores, variables como: el nivel de exposición a la situación del 7 de julio, experiencia anterior en relación con el terrorismo, incertidumbre respecto a su seguridad en esos momentos... jugaron un papel determinante en la percepción de amenaza entre la población londinense. Concluyen que la preparación para el terrorismo puede reducir su impacto en la población.

En el atentado del 11 de marzo en Madrid (Lillo et al., 2004), aunque no había una preparación para un atentado terrorista de ese calibre, los aspectos informativos cobraron una gran importancia en la gestión de la atención psicológica a las victimas y en la coordinación interinstitucional

Una investigación (Schütz \& Wiedemann, 2000) realizada respecto a la compañía farmacéutica alemana Boehringer Mannheim, puede ayudar a entender la importancia de la información previa a la situación de emergencia para mejorar la confianza y la credibilidad. Se facilitó a las personas de la zona un folleto de 4 páginas sobre información de emergencias y se entrevistó posteriormente a personas que habian recibido la información y que no la habían recibido. Las personas que recibieron la información eran capaces de especificar con mayor frecuencia la conducta adecuada, aunque las diferencias sólo fueron esta- disticamente significativas en un caso (encerrarse en una habitación); las diferencias eran más claras en cuanto a la conducta inadecuada en esos casos. Cuando se les preguntó por la confianza y credibilidad de la empresa, las personas que recibieron la información, tenian mayor confianza en los esfuerzos de la compañía farmacéutica para reducir riesgos (las diferencias eran estadísticamente significativas).

Además, el uso de modelos de actuación (como el Modelo de Intervención psicosocial para la información telefónica en situaciones de emergencia de Muñoz y Álvarez, 2000) puede facilitar la gestión de las necesidades informativas y tiene innegables ventajas.

La eficacia de las acciones informativas está condicionada también por las relaciones interinstitucionales y por la concepción y el tratamiento que tenga cada uno de la emergencia (De Miguel y Muñoz, 1998).

Es fundamental reconocer la importancia del intercambio de información y de la comunicación en la gestión de desastres; tampoco hay que olvidar el papel de apoyo que ejercen las redes integradas en la propia población, en la prevención de desastres que las afectan (Anderson, 1997). Además los responsables técnicos de telecomunicaciones tienen una gran responsabilidad porque en situaciones de catástrofe se convierten en un recurso importante para mitigar las consecuencias de la misma. Mileti y Peek (2000) apuntan que uno de los mejores predictores de una buena comunicación en situaciones de emergencia es la calidad de las relaciones interpersonales en momentos en los que no hay crisis; porque la gente que se conoce trabaja mejor en momentos de crisis (Mileti \& Sorensen, 1987). Por este motivo, una manera de promover una comunicación 
fluida es la realización de simulacros y ejercicios en el lugar donde podría tener lugar la emergencia.

Otro aspecto que no debemos olvidar es la gestión de las falsas alarmas. Sólo constituyen un problema cuando se gestionan mal (se ocultan las mismas, se realizan varias alarmas innecesarias en un breve periodo de tiempo, hay deficiente manejo de la situación con los medios de comunicación social) porque originan pérdida de credibilidad entre la población. Para que no afecten negativamente hay que tratar de explicar a la población por qué han ocurrido; además esto puede incrementar la consciencia de riesgo entre la población diana (Breznitz $\&$ Wolf, 1984)

Según Barnes, Hayden, Schultz \& Benight, (2006) las falsas alarmas pueden crear un mayor nivel de vigilancia si hay un entendimiento del evento y de las razones de la alerta; además proporcionan una oportunidad de aprender las respuestas apropiadas para futuras situaciones y de poner a prueba los sistemas de alerta, respuestas y protocolos.

Hay otros estudios que en relación a las falsas alarmas plantean la necesidad de usar información adicional para tomar medidas protectoras desde todos los ámbitos y prevenir posibles conflictos en el futuro (Zabyshny \& Ragland, 2003)

Algunos autores (De Miguel y Muñoz, 1998) plantean que a lo largo de todo el proceso debe haber una sola cabeza coordinadora de la intervención, sobre todo cuando haya un alto número de afectados e instituciones. Una situación puede complicarse cuando se dan mensajes desde varios ámbitos; las decisiones y la responsabilidad asociada deben asumirse desde un solo órgano coordinador, para evitar difusión de responsabili- dades y facilitar que las acciones se realicen de manera más rápida y eficaz.

El nivel de información que requiere la población en cada momento es variable: la disponibilidad de conocimientos sobre la emergencia decrece a medida que pasa el tiempo (Mossman et al., 1990; Van Eijndhoven et al., 1994; Schütz \& Wiedemann, 2000) y es necesario adaptarse a ello.

\section{PAUTAS DE TRANSMISIÓN DE INFORMACIÓN A LA POBLACIÓN}

Las personas necesitan información en una emergencia; este es un fenómeno universal que se ha comprobado en varias ocasiones en países como Francia (Lalo, 1990), Portugal (Nicolau, 1990) e Italia (De Marchi, 1991).

Proporcionar información sobre potenciales riesgos es un derecho de la población (Schütz \& Wiedemann, 2000). La información es una herramienta fundamental para manejar la situación de crisis o emergencia que se ha desencadenado. Se trata, según Fidalgo (2006b) de que la información se convierta en un elemento activo del plan de emergencia en esas situaciones difíciles. Sin información la cooperación de las personas se hace difícil. La comunicación de los riesgos en una emergencia puede ayudar a la gente a afrontar la situación, tomar decisiones respecto a los riesgos y sacar provecho de los mismos y comenzar lo antes posible el retorno a sus vidas cotidianas (ORAU, 2006).

También es cierto que no se puede dar toda la información, por varias razones: por seguridad, porque sería inoperativo proporcionar toda la información a todos los implicados, porque consumiría mucho tiempo innecesariamente... Según Pérez de Tudela (1994) los límites 
de la información en situaciones socialmente prioritarias están en la propia autocritica, en la existencia de riesgos que pueden amenazar la paz social y en la posibilidad de que la misión informativa pudiera estar distorsionada.

Los mensajes deben ser simples, breves y deben transmitir ante todo seguridad. Hay que tener en cuenta que la información debe ser escuchada por la población, o por lo menos recibir una señal que les alerte de la presencia de un peligro; el que la persona escuche esta señal depende de las condiciones ambientales (Lindel y Perry, 1992). Por ejemplo, no se podrán oír sirenas o campanas de alerta, si hay un fuerte viento o las personas disponen en su domicilio de potentes aparatos de aire acondicionado, o cualquier otro dispositivo que pueda interferir estas señales.

Robles y Medina (2002) añaden que la información debe ser objetiva y desdramatizante en lo que se refiere a los peligros reales de la situación de emergencia, las medidas de protección y lo que debe y no hacerse en caso de producirse la emergencia o de evolucionar de una manera imprevisible. Los mensajes deben posibilitar acciones, diciendo lo que puede hacerse; de esta manera se evitan reacciones cercanas al pánico que no son frecuentes en estos casos.

En opinión de Mileti y Peek (2000) que la población entienda la información que se le facilita, implica que las personas tengan percepción de riesgo y personalicen el riesgo, es decir que interpreten que ese riesgo puede afectarles en un breve periodo de tiempo. Después las personas deciden qué van a hacer respecto al riesgo percibido y deben llevar a cabo la conducta elegida.

En una situación de emergencia es fundamental saber de quién se puede obtener información para que el progreso de la misma se haga de manera adecuada y a quién debe proporcionarse la información, para facilitar la gestión de todo el proceso. En muchos casos las mismas personas asumen los dos roles; entonces debemos velar por que la comunicación sea fluida entre ellos. Según Pérez de Tudela (1994) ni las declaraciones de testigos presenciales de la emergencia ni la información técnica, son suficientes para que la población proceda con prontitud. Además a veces

Tabla 2. Requisitos de información para lograr mayor eficacia en los mensajes (Pérez de Tudela, 1994; Muñoz, 2000; Mileti \& Peek, 2000; Marín, 2005; Fidalgo 2006b, Bourque, 2006)

\begin{tabular}{l} 
REQUISITOS DE LOS MENSAJES PARA LOGRAR EFICACIA \\
- Credibilidad (Confianza en las fuentes) \\
- Claridad (Expresión del mensaje en términos sencillos) \\
- Continuidad (repetición con frecuencia, sin que ésta sea excesiva) \\
- Coherencia (tener pleno sentido para quien recibe la información) \\
- Adecuación (tener en cuenta costumbres, nivel sociocultural...) \\
- Viabilidad (utilizar cauces adecuados) \\
- Accesibilidad (usar los canales de información habituales) \\
- Comprensividad (lenguaje sin tecnicismos innecesarios) \\
- Coordinación (acuerdo entre las fuentes, evitando contradicciones) \\
- Familiaridad (conocimiento de la fuente que emite el mensaje) \\
\hline
\end{tabular}


Tabla 3. Proveedores y destinatarios de la información en situaciones de emergencia

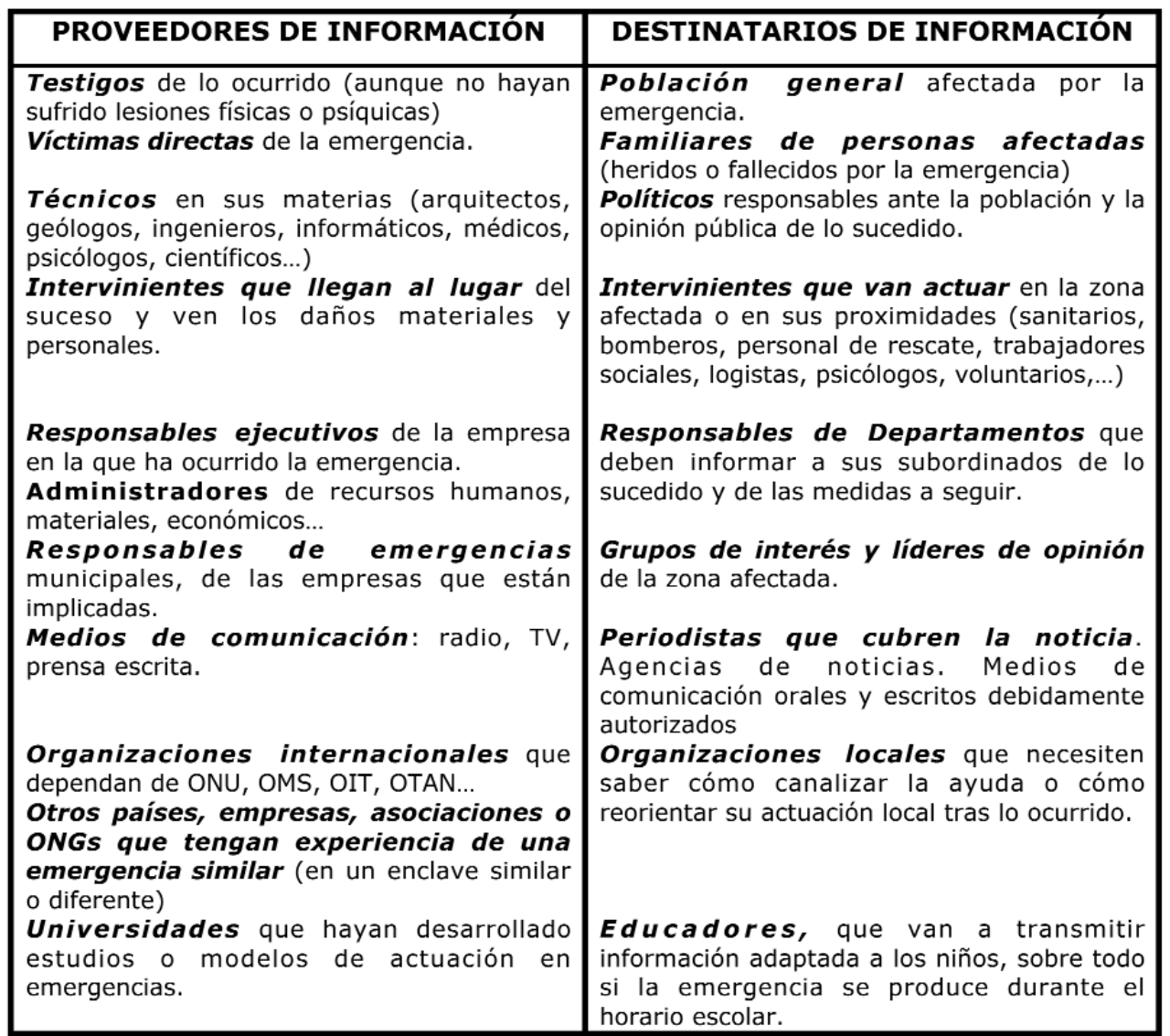

los mensajes que se dan a la población son contradictorios o se interpretan de manera ambigua.

Otro aspecto en el que hay que hacer hincapié es la necesidad de recabar feedback de proveedores y de destinatarios de la información, para que el proceso comunicativo se realice adecuadamente. En una investigación realizada por Glik, Harrison, Davoudi y Riopelle en 2004 (Citado por Bourque, 2006), donde 93 personas fueron entrevistadas respecto de dónde obtendrían información ante un eventual ataque bioterrorista por toxina botulinica, las personas dijeron recurrir a radio y televisiones locales y nacionales, internet, servicios de salud de la comunidad... sin embargo no eligieron políticos ni tan siquiera médicos.

Así la respuesta protectora de la gente ante alertas es consecuencia de las percepciones que se forman inmediatamente antes de realizar las acciones que se requieren; es decir, si perciben que son apropiadas, considerando las circunstancias de peligro inminente (Mileti \& Peek, 2000). Las percepciones que se forman las personas en emergencias siguen 
Tabla 4. Ejemplos de mensajes dirigidos a la población en situación de emergencia

\section{MENSAJES A LA POBLACIÓN AFECTADA POR LA EMERGENCIA}

(Altavoces de un centro comercial). Atención señores clientes.

Les habla la Dirección del centro Comercial XXXX. Se ha producido el desplome de una de las alas de nuestro edificio y hay un incendio en la planta 5. La situación está siendo controlada y se están desplazando a este lugar recursos de seguridad suficientes.

Por su seguridad, vamos a proceder a la evacuación del edificio. Dirijase a la puerta más próxima marcada con una luz roja, de manera ordenada, y nuestros empleados le indicarán el camino de salida.

Cuando esté fuera del edificio vaya a la calle YYY donde hay instalada una carpa donde tomarán sus datos y podrán ayudarle en lo que usted necesite.

(Mensaje del teléfono de información del 112) Ha llamado al teléfono de información especial habilitado por la Dirección del Centro de Coordinación de emergencias del $112 \mathrm{de}^{* * * *}$.

Se ha producido un vertido tóxico de Plomo al río ${ }^{* * * *}$, como consecuencia del mismo el agua corriente está contaminada. La situación es grave.

Las autoridades competentes están avisadas y están realizando acciones para garantizar el consumo saludable de agua en el menor tiempo posible.

No abra el grifo de su domicilio, use agua embotellada para beber y cocinar, vigile que niños y personas mayores sigan estas recomendaciones.

Si ya ha consumido agua del grifo o la ha usado para ducharse o beber y nota los siguientes síntomas: dolor de cabeza, picores en la piel, malestar gástrico... llame al 112 o espere el final de esta locución y un operador sanitario le atenderá.

Esta información se actualiza cada 30 minutos, la última actualización se ha producido a las 22:10 horas.

Nota: Cada punto y aparte es una pausa

el mismo proceso que en cualquier otro evento social; si la información que se les proporciona ayuda a focalizar la atención en lo esencial o urgente en esas circunstancias, se favorecerá una actuación adecuada y reducirá la ansiedad de ejecución que aparece en estas situaciones.

La emergencia puede evolucionar de manera que sea necesario introducir cambios en la información facilitada a la población o simplemente actualizarla. Los cambios deben establecerse de manera ordenada, teniendo siempre en cuenta la información facilitada con anterioridad y previendo los mecanismos de comunicación necesarios (Pérez de Tudela, 1994; Muñoz 2000): 
Información a la población en situaciones de emergencia y riesgo colectivo

1. Realizar los cambios en los mensajes regulares, periódicos que la población sabe que van a transmitirse. Esto produce menos distorsión y mayor confianza en las Instituciones y Servicios de Emergencia. Si se emite un mensaje y luego se reformula de manera muy diferente puede originar confusión.

2. Informar con la mayor antelación posible a todos los equipos intervinientes (antes incluso de su difusión a la población afectada), porque sino se podría producir un efecto de desajuste y desinformación en los profesionales que se enteran de los cambios por medios de comunicación y no a través de sus centros de coordinación o superiores jerárquicos.

3. Se primará el acceso a estos cambios de noticias, según el tipo de información novedosa que sea necesario proporcionar. Debe garantizarse que la información llegue a toda la población de la manera más rápida y segura posible.

4. La información debe llegar al mismo tiempo a todas las poblaciones afectadas. Cuando un grupo recibe antes que otro el mensaje, pueden producirse efectos de desestabilización social, intentos de pequeñas migraciones rápidas, situaciones peligrosas difíciles de controlar.

En todos los casos hay que asegurarse de que la población accede a toda la información de manera homogénea, buscando los medios para que esto sea así. Es conveniente repetir los mensajes, sobre todo al principio, para reducir el riesgo de malas interpretaciones y asegurarse la buena comprensión (Mikami \& Ikeda, 1985).

\section{CONCLUSIONES}

1. Sería recomendable la formación en aspectos psicosociales (conductuales, cognitivos, afectivos, de interacción social, etc.) de la información en grandes emergencias, a los portavoces de organismos públicos y privados, así como a responsables que tienen personal a su cargo a los que puedan tener que proporcionarles información en emergencias.

2. La gestión de una situación de emergencia no puede realizarse sin una adecuada coordinación interinstitucional y un conocimiento previo de la población a la que se dirige la información.

3. Proporcionar información de manera previa a la situación de emergencia tiene un efecto positivo en la gestión informativa de la emergencia y en la confianza y credibilidad de la población en la empresa 0 persona que emite el mensaje.

4. Es sumamente útil informar a la población, especialmente a la población local, respecto a los riesgos de accidentes y las precauciones que debe contemplar, porque se consiguen comportamientos más adaptados a las circunstancias y menos conductas dificiles de manejar.

5. El manejo de la información en los momentos inmediatamente posteriores a la situación de emergencia va a influir de una manera sustancial en la repercusión pública, en las percepciones de la personas afectadas por la misma en cuanto a la causa 0 responsabilidad del mismo y en el comportamiento que muestren en la emergencia (tanto en los primeros 
momentos, como ante situaciones de emergencia posteriores de características comparables).

6. La preparación o elaboración de un plan de actuación coordinado y el diseño previo (en situación de normalidad) de algunos modelos de mensajes que podría ser necesario difundir entre la población, puede facilitar la gestión acorde a la situación, cuando se produce la emergencia.

7. La realización de simulacros de actuación con cierta periodicidad permitiria preparar a la población afectada, con mayores garantías de eficacia y efectividad, especialmente en lugares o zonas "sensibles" a este tipo de eventos

8. La gestión informativa y de riesgos debe centrarse en minimizar conductas de riesgo en la situación de emergencia, mediante un manejo certero de la información que se proporciona y de los momentos en los que se facilita.

9. Las falsas alarmas que pueden producirse en la activación de planes de emergencia, pese a los lógicos inconvenientes que generan, pueden ser útiles como un mejor desenvolvimiento en situaciones auténticas en el futuro y para incrementar la percepción de riesgo entre la población expuesta a los previsibles riesgos.

10. Muchas de las grandes emergencias en que ha habido riesgo colectivo han cambiado la legislación, o la gestión de situaciones de emergencia. Deben realizarse más estudios para implantar cambios de manera proactiva y no esperar a que una catástrofe natural o artificial obligue a modificar las directrices vigentes.

\section{BIBLIOGRAFÍA}

Acinas, M.P. (2007). Gestión de la información y mensajes a la población en situaciones de emergencia, evacuaciones y simulacros. Emergencia, 19, $88-95$

Anderson, P.S. (1997). Adaptación de los nuevos sistemas de comunicación a la prevención de desastres en zonas urbanas. Stop Disasters, 32 (2), 11-14.

Barnes, L.R., Gruntfest, E.C., Hayden, M.H., Schultz, D.M. y Benight, C. (2006). False Alarms and Close Calls: A Conceptual Model of Warning Accuracy. Weather and Forecasting. Forecasters Forum 5 September 2006. Extraido el 29 de noviembre de 2007 desde $h t t p: / / w e a t h e r c o n f e r-$ encedinner.googlepages.com/Barnes_etal_submitted_revisions12_18.pdf

Bourque, L.B. (2006). The disaster-Public Health Nexus. Center for Public Health and Disasters. University of California. Los Ángeles. Extraído el 2 de septiembre de 2006 desde http://dels. nas.edu/dr/docs/dr11/bourque.ppt
Breznitz, S. y Wolf, C. (1984). The Psychology of False Alarms. Erlbaum Associates, Hillsdale, NI

CEISE (1989) Seminario Internacional sobre los problemas de la Información a la población en caso de emergencia: El caso particular de una autoevacuación colectiva. Centro Europeo de Investigación Social de Situaciones de Emergencia CEISE. Conferencia Virtual. Seminario Internacional. Dirección General de Protección Civil España. Extraído el 01 de diciembre de 2007 desde $h t t p: / / w w w . p r o-$ teccioncivil.org/ceise/ceise1989.htm\#p2

Cortés, B. (2002). Desastres y procesos psicosociales. Desde la crisis en la gestion hacia la gestión de las crisis. CEISE. Protección Civil España. Extraído el 13 de agosto de 2006 desde http://www.proteccioncivil.org/ceise/tesis2002/tesispc2002_2.pdf

Fernández Millán, J.M. (Coord.) (2005). Apoyo psicológico en situaciones de emergencia. Madrid: Pirámide.

Fidalgo, M (2006a). Nota Técnica de Prevención 
390: La conducta humana ante situaciones de emergencia: Análisis de proceso en la conducta. Ministerio de Trabajo y Asuntos Sociales. Instituto Nacional de Seguridad e Higiene en el Trabajo. Extraído el 12 de agosto de 2006 desde www.mtas. es/insht/ntp/ntp_390.htm

Fidalgo, M (2006b). Nota Técnica de Prevención 395: La conducta humana ante situaciones de emergencia: la conducta colectiva. Ministerio de Trabajo y Asuntos Sociales. Instituto Nacional de Seguridad e Higiene en el Trabajo Extraido el 12 de agosto de 2006 desde www.mtas.es/insht/ntp/ ntp_395.htm

HM Government, (n.d.). Preparing for emergencies. What you need to know. Extraido el 19 de agosto de 2005 desde www.preparingforemergencies.gov.uk

Lalo, A. (1990). Informing the public on major technological risks: Communication strategies of the Bouches-du-Rhône campaign, April-June 1989. In H.B.F. Gow yH. Otway (Eds). Communicat ing of public about major accident hazards (204231). London: Elsevier Science Publishers.

Latané, B. y Darley, J. (1974). Determinantes sociales de la intervención del espectador. En J. R. Torregrosa (Ed.), Teoría e investigación en la psicología social actual (261-274). Madrid: Instituto de Opinión Pública (Orig. 1970).

Lillo, A., Muñoz, F.A., Parada, E., Puerta, A., Ramos, M., Pereira, M., Del Val, M. y Guijarro, A. (2004) Intervención Psicológica tras los atentados del 11 de Marzo en Madrid. Clínica y Salud, 15, 7-88.

Lindel, M.K. y Perry, R.W. (1992). Behavioral foundations of Community Emergency Planning. Hemisphere Publishing, Washington, DC.

Marin, F. (2005). Gestión técnica y de la comunicación en situaciones especiales. Crisis, emergencias y negociación. Madrid: Fragua.

De Marchi, B. (1991). The Seveso Directive: An Italian pilot study in enabling communication. Risk Analysis, 11 (2), 207-215.

Miguel A. De y Muñoz F.A. (1998). Aspectos conceptuales de la Intervención en Crisis. Revista de Psiquiatria de la Universidad de Barcelona, 25 (6), 177-181.

Mikami, S. y Ikeda, K. (1985). International Journal of Mass Emergencies and Disasters 3, 107.
Mileti, D.S. y Sorensen, J.H. (1987). In Weinstein (Ed.) Taking care: Why people take precautions. Cambridge Univ. Press. Cambridge, MA.

Mileti, D. S. y Peek, L. (2000). The social psychology of public response to warnings of nuclear power plant accident. Journal of Hazardous Materials, 75 (2000), 181-194. Extraido el 2 de septiembre de 2006 desde http://archone.tamu edu/hrrc/publications/pdf/00-13A.pdf

Mossmann, G.K., Follows, A., Fisher, R.W. y Humphreys, D. (1990). Control of industrial major accident hazards (CIMAH) regulations - information to public: A local authority emergency planning perspective. In H.B.F., Gow yH. Otway (Eds). Communicating with the public about major accident hazards (96-102). London: Elsevier Science Publishers.

Muñoz, F.A. (2000). Aspectos psicosociales, de la información a la población en emergencias. Revista Intervención Psicosocial, 9 (3), 371-377.

Muñoz, F.A. y Álvarez A.J. (2000). Programa de intervención psico-social para la información a la población en emergencias masivas y catástrofes. Emergencias, (12), 42-46.

Nicolás, L. De, Artetxe, A.I., Jáuregui, A., López, $\mathrm{S}$. (2000). Intervención psicológica en situaciones de emergencia y desastres. Vitoria: Servicio de Publicaciones del Gobierno Vasco. 2000.

Nicolau, J.A. (1990). Public information for major industrial accidents. In H.B.F. Gow yH. Otway (Eds). Communicating of public about major accident hazards (204-231). London: Elsevier Science Publishers.

ORAU (n.d.). Crisis and emergency risk communication. Oak Ridge Associated Universities (ORAU). Oak Ridge Institute for Science and Education (ORISE). EE.UU. Extraído el 2 de septiembre de 2006 desde http://www.orau.gov/cdcynergy /erc/CERC\%20Course\%20Materials/Instructor\%20P PT\%20Slides/Overview.ppt

Pérez de Tudela, C. (1994). La información en las catástrofes. Madrid: Fundación MAPFRE.

Robles, J.I. y Medina, J.L. (2002). Intervención psicológica en las catástrofes. Madrid: Sintesis.

Rubin, G.J., Brewin, C.R., Greenberg, N., Simpson, J. y Wessely, S. (2005). Psychological and behavioural reactions to the bombings in London on 7 July 2005: cross sectional survey of a representative sam- 
ple of Londoners. BMJ Publishing Group Ltd. British Medical Association. BMJ, 331, 606. Extraído el 5 de septiembre de 2006 desde http://bmj. bmijournals.com/cgi/content/full/331/7517/606.

San Juan, C. (Ed.) (2001). Catástrofes y ayuda en emergencia. Estrategias de evaluación, prevención y tratamiento. Barcelona: Icaria - Antrazyt.

Schuster, M.A., Stein, B.D., Jaycox, L.H., Collins, R.L., Marshall, G.N., Elliot, M.N. et al. (2001). A Nacional survey of stress reactions alter the september 11, 2001, Terrorist attacks. New England Journal Medicine, 345, 1507-1512.

Schütz, H. y Wiedemann, P.M. (2000). Hazardous Incident Information for the Public: Is it Useful? The Australasian Journal of Disaster and Trauma Studies, 2000-2. Special Edition: Risk Information yCommunication. Extraído el 1 de septiembre de 2006 desde http://www.massey.ac.nz/ trauma/issues/2000-2/schuetz.htm

Slaikeu, K.A. (Coord) (1996). Intervención en crisis: Manual para la práctica y la investigación. México: El Manual Moderno.
Sorensen, J.H., Vogt, B.M. y Mileti, D.S. (1987). Evacuation: An assessment of Planing and Research, Report ORNL- 6376, Oak Ridge National Laboratory, Oak Ridge TN.

Turner, R.H., Nigg, J.M.,Paz, D. y Young, B.S. (1981). Community Response to Earthquake Threats in Southern California. University of California Press. Los Angeles.

Turner, R.H., Nigg, J.M. y Paz, D. (1986). Waiting for Disaster: Earthquake match in California. University of California Press. Berkeley, CA.

Van Eijndhoven, J.C.M., Weterings, R.A.P.M., Worrell, C.W. et al. (1994). Risk communication in The Nederlands: The Monitored introduction of EC "post Seveso" Directive. Risks Analysis, 14, 87-96.

Zabyshny, A.A. y Ragland, D.R. (2003) False Alarms and Human - Machine Warning Systems. UC. Berkeley Traffic Safety Center. Institute of Transportation Studies. University of California. Berkeley. Extraído el 29 de noviembre de 2007 desde http://repositories. cdlib.org/cgi/viewcontent.cgi?article $=1005 \&$ context $=$ its $/$ tsc 